\title{
Connes-amenability and normal, virtual diagonals for measure algebras, I
}

\author{
Volker Runde* \\ Dedicated to the memory of Barry E. Johnson, \\ 1937-2002, \\ on whose shoulders many of us stand.
}

\begin{abstract}
We prove that the measure algebra $M(G)$ of a locally compact group $G$ is Connesamenable if and only if $G$ is amenable.
\end{abstract}

Keywords: locally compact group; group algebra; measure algebra; amenability; Connes-amenability; normal, virtual diagonal.

2000 Mathematics Subject Classification: 22D15, 43A10 (primary), 43A20, 43A60, 46E15, 46H25, 46M20, 47B47.

\section{Introduction}

In [16], B. E. Johnson introduced the notion of an amenable Banach algebra, and proved that a locally compact group $G$ is amenable if and only if its group algebra $L^{1}(G)$ is amenable. The theory of amenable Banach algebras has been a very active field of research ever since. Once of the deepest results in this theory is due to A. Connes ([5]; see also [2]) and U. Haagerup ([12]): A $C^{*}$-algebra is amenable if and only if it is nuclear. In [24], S. Wassermann showed that a von Neumann algebra is nuclear/amenable if and only if it is subhomogeneous (see [22] for a proof that avoids the nuclearity-amenability nexus). This suggests that the definition of amenability from [16] has to be modified to yield a sufficiently rich theory for von Neumann algebras.

A variant of that definition - one that takes the dual space structure of a von Neumann algebra into account - was introduced in [18], but is most commonly associated

\footnotetext{
${ }^{*}$ Research supported by NSERC under grant no. 227043-00.
} 
with A. Connes' paper [4]. For this reason, we refer to this notion of amenability as to Connes-amenability (the origin of this name seems to be A. Ya. Helemski1̌'s paper [13]). As it turns out, Connes-amenability is the "right" notion of amenability for von Neumann algebras: It is equivalent to several other important properties such as injectivity and semidiscreteness ([2], [四], [5], [9], [25]; see [23, Chapter 6] for a self-contained exposition).

The definition of Connes-amenability makes sense for a larger class of Banach algebras (called dual Banach algebras in [22]). Examples of dual Banach algebras (other than $W^{*}$-algebras) are: $\mathcal{B}(E)$, where $E$ is a reflexive Banach space; $M(G)$, where $G$ is a locally compact group; $P M_{p}(G)$, where $p \in(1, \infty)$ and $G$ is a locally compact group (these algebras are called algebras of $p$-pseudomeasures). The investigation of Connes-amenability for dual Banach algebras which are not $W^{*}$-algebras is still in its initial stages. Some results on Connes-amenable $W^{*}$-algebras, carry over: For instance, in [20], A. T.-M. Lau and A. L. T. Paterson showed that, for an inner amenable group $G$, the group von Neumann algebra $\operatorname{VN}(G)=P M_{2}(G)$ is Connes-amenable if and only if $G$ is amenable; this is generalized to $P M_{p}(G)$ for arbitrary $p \in(1, \infty)$ in [22]. On the other hand, one cannot expect matters for general dual Banach algebras to turn out as nicely as for von Neumann algebras: In [22], it was shown that $\mathcal{B}(E)$ is not Connes-amenable if $E=\ell^{p} \oplus \ell^{q}$ with $p, q \in(1, \infty) \backslash\{2\}$ and $p \neq q$.

The dual Banach algebra we are concerned with in this paper is the measure algebra $M(G)$ of a locally compact group $G$. As for von Neumann algebras, amenability in the sense of [16] is too strong a notion to deal with measure algebras in a satisfactory manner: In [7], H. G. Dales, F. Ghahramani, and A. Ya. Helemskiù prove that $M(G)$ is amenable for a locally compact group $G$ if and only if $G$ is discrete and amenable. In contrast, Connes-amenability is a much less restrictive demand: Since the amenability of

a locally compact group $G$ implies the amenability of $L^{1}(G)$, and since $L^{1}(G)$ is $w^{*}$-dense in $M(G)$, it follows easily that $M(G)$ is Connes-amenable provided that $G$ is amenable. In this paper, we prove the converse.

I am grateful to S. Tabaldyev for discovering a near fatal error in an earlier, stronger version of Lemma 4.2.

\section{Connes-amenability and normal, virtual diagonals}

This section is preliminary in character. We collect the necessary definitions we require in the sequel. All of it can be found in [22], but sometimes our choice of terminology here is different.

Let $\mathfrak{A}$ and $\mathfrak{B}$ be Banach algebras, and let $E$ be a Banach $\mathfrak{A}-\mathfrak{B}$-bimodule. Then $E^{*}$ becomes a Banach $\mathfrak{B}$-A-bimodule via

$$
\langle x, b \cdot \phi\rangle:=\langle x \cdot b, \phi\rangle \quad \text { and } \quad\langle x, \phi \cdot a\rangle:=\langle a \cdot x, \phi\rangle \quad\left(a \in \mathfrak{A}, b \in \mathfrak{B}, \phi \in E^{*}, x \in E\right) .
$$


Definition 1.1 Let $\mathfrak{A}$ and $\mathfrak{B}$ be Banach algebras. A Banach $\mathfrak{A}-\mathfrak{B}$-bimodule $E$ is called dual if there is a closed submodule $E_{*}$ of $E^{*}$ such that $E=\left(E_{*}\right)^{*}$.

Remark There is no reason, in general, for $E_{*}$ to be unique. If we refer to the $w^{*}$-topology on a dual Banach module $E$, we always mean $\sigma\left(E, E_{*}\right)$ with respect to a particular, fixed (often obvious) predual $E_{*}$.

The following definition is due to B. E. Johnson ([16]):

Definition 1.2 A Banach algebra $\mathfrak{A}$ is called amenable if, for every dual Banach $\mathfrak{A}$ bimodule $E$, every bounded derivation $D: \mathfrak{A} \rightarrow E$ is inner.

We are interested in a particular class of Banach algebras:

Definition 1.3 A Banach algebra $\mathfrak{A}$ is called dual if it is dual as a Banach $\mathfrak{A}$-bimodule.

Remark A Banach algebra which is also a dual space is a dual Banach algebra if and only if multiplication is separately $w^{*}$-continuous.

Examples 1. Every $W^{*}$-algebra is dual.

2. If $E$ is a reflexive Banach space, then $\mathcal{B}(E)=\left(E \hat{\otimes} E^{*}\right)^{*}$ is dual.

3. If $G$ is a locally compact group, then $M(G)=\mathcal{C}_{0}(G)^{*}$ is dual.

4. If $\mathfrak{A}$ is an Arens regular Banach algebra, then $\mathfrak{A}^{* *}$ is dual.

The following choice of terminology is motivated by the von Neumann algebra case:

Definition 1.4 Let $\mathfrak{A}$ and $\mathfrak{B}$ be dual Banach algebras. A dual Banach $\mathfrak{A}-\mathfrak{B}$-bimodule is called normal if, for each $x \in E$, the maps

$$
\mathfrak{A} \rightarrow E, \quad a \mapsto a \cdot x
$$

and

$$
\mathfrak{B} \rightarrow E, \quad b \mapsto x \cdot b
$$

are $w^{*}$-continuous.

We can now define Connes-amenable, dual Banach algebras:

Definition 1.5 A dual Banach algebra $\mathfrak{A}$ is called Connes-amenable if, for every normal, dual Banach $\mathfrak{A}$-bimodule $E$, every bounded, $w^{*}$-continuous derivation $D: \mathfrak{A} \rightarrow E$ is inner. 
Amenability in the sense of [16], can be intrinsically characterized in terms of so-called approximate and virtual diagonals ([17]). There is a related notion for Connes-amenable, dual Banach algebras.

If $E_{1}, \ldots, E_{n}$ and $F$ are dual Banach spaces, we write $\mathcal{L}_{w^{*}}\left(E_{1}, \ldots, E_{n} ; F\right)$ for the bounded, separately $w^{*}$-continuous, $n$-linear maps from $E_{1} \times \cdots \times E_{n}$ into $F$. In case $E_{1}=\cdots=E_{n}=: E$, we simply let $\mathcal{L}_{w^{*}}^{n}(E, F):=\mathcal{L}_{w^{*}}\left(E_{1}, \ldots, E_{n} ; F\right)$.

Let $\mathfrak{A}$ and $\mathfrak{B}$ be Banach algebras. Then $\mathfrak{A} \hat{\otimes} \mathfrak{B}$ becomes a Banach $\mathfrak{A}$ - $\mathfrak{B}$-bimodule via

$$
a \cdot(x \otimes y):=a x \otimes y \quad \text { and } \quad(x \otimes y) \cdot b:=x \otimes y b \quad(a, x \in \mathfrak{A}, b, y \in \mathfrak{B}) .
$$

Suppose that $\mathfrak{A}$ and $\mathfrak{B}$ are dual. It is then routinely checked that $\mathcal{L}_{w^{*}}^{2}(\mathfrak{A}, \mathfrak{B} ; \mathbb{C})$ is a closed $\mathfrak{B}$-Â-submodule of $(\mathfrak{A} \hat{\otimes} \mathfrak{B})^{*}$.

Let $\mathfrak{A}$ be a dual Banach algebra, and let $\Delta_{\mathfrak{A}}: \mathfrak{A} \hat{\otimes} \mathfrak{A} \rightarrow \mathfrak{A}$ denote the diagonal operator induced by $\mathfrak{A} \times \mathfrak{A} \ni(a, b) \mapsto a b$. Since multiplication in $\mathfrak{A}$ is separately $w^{*}$-continuous, we have $\Delta_{\mathfrak{A}^{*}}^{*} \mathfrak{A}_{*} \subset \mathcal{L}_{w^{*}}^{2}(\mathfrak{A}, \mathbb{C})$. Taking the adjoint of $\left.\Delta_{\mathfrak{A}}^{*}\right|_{\mathfrak{A}_{*}}$, we may thus extend $\Delta_{\mathfrak{A}}$ to $\mathcal{L}_{w^{*}}^{2}(\mathfrak{A}, \mathbb{C})^{*}$ as an $\mathfrak{A}$-bimodule homomorphism (we denote this extension by $\Delta_{w^{*}}$ ).

Definition 1.6 Let $\mathfrak{A}$ be a dual Banach algebra. An element $M \in \mathcal{L}_{w^{*}}^{2}(\mathfrak{A}, \mathbb{C})^{*}$ is called a normal, virtual diagonal for $\mathfrak{A}$ if

$$
a \cdot \mathrm{M}=\mathrm{M} \cdot a \quad \text { and } \quad a \Delta_{w^{*}} \mathrm{M}=a \quad(a \in \mathfrak{A}) .
$$

One connection between Connes-amenability and the existence of normal, virtual diagonals is fairly straightforward ([8], [6]): If $\mathfrak{A}$ has a normal, virtual diagonal, then $\mathfrak{A}$ is Connes-amenable; in fact, it implies a somewhat stronger property $([22])$. The main problem with proving the converse is that, in general, the dual module $\mathcal{L}_{w^{*}}^{2}(\mathfrak{A}, \mathbb{C})^{*}$ need not be normal. For von Neumann algebras, however, Connes-amenability and the existence of normal, virtual diagonals are even equivalent ([8], [10]). We suspect, but have been unable to prove - except in the discrete case - that the same is true for the measure algebras of locally compact groups.

\section{Separately $\mathcal{C}_{0}$-functions on locally compact Hausdorff spa- ces}

Our notation is standard: For a topological space $X$, we write $\mathcal{C}_{b}(X)$ for the bounded, continuous functions on $X$; if $X$ is locally compact and Hausdorff, $\mathcal{C}_{0}(X)$ (or rather $\mathcal{C}(X)$ if $X$ is compact) denotes the continuous functions on $X$ vanishing at infinity, and $M(X) \cong \mathcal{C}_{0}(X)^{*}$ is the space of regular Borel measures on $X$.

Let $X$ and $Y$ be locally compact Hausdorff spaces. In this section, we give a description of $\mathcal{L}_{w^{*}}(M(X), M(Y) ; \mathbb{C})$ as a space of separately continuous functions on $X \times Y$. 
Definition 2.1 Let $X$ and $Y$ be locally compact Hausdorff spaces. A bounded function $f: X \times Y \rightarrow \mathbb{C}$ is called separately $\mathcal{C}_{0}$ if:

(a) for each $x \in X$, the function

$$
Y \rightarrow \mathbb{C}, \quad y \mapsto f(x, y)
$$

belongs to $\mathcal{C}_{0}(Y)$;

(b) for each $y \in Y$, the function

$$
X \rightarrow \mathbb{C}, \quad x \rightarrow f(x, y)
$$

belongs to $\mathcal{C}_{0}(X)$.

We define $\mathcal{S C}_{0}(X \times Y)$ as the collection of all separately $\mathcal{C}_{0}$-functions.

Lemma 2.2 Let $X$ and $Y$ be locally compact Hausdorff spaces, and let $f \in \mathcal{S C}_{0}(X \times Y)$. Then the following hold:

(i) for each $\mu \in M(X)$, the function

$$
Y \rightarrow \mathbb{C}, \quad y \mapsto \int_{X} f(x, y) d \mu(x)
$$

belongs to $\mathcal{C}_{0}(Y)$;

(ii) for each $\nu \in M(Y)$, the function

$$
X \rightarrow \mathbb{C}, \quad x \mapsto \int_{Y} f(x, y) d \nu(y)
$$

belongs to $\mathcal{C}_{0}(X)$.

Proof We only prove (i).

Let $\mu \in M(X)$. Since the measures with compact support are norm dense in $M(X)$, there is no loss of generality if we suppose that $X$ is compact. Suppose that $Y$ is not compact (the compact case is easier), and let $Y_{\infty}$ be its one-point-compactification. Extend $f$ to $X \times Y_{\infty}$ by letting

$$
f(x, \infty)=0 \quad(x \in X),
$$

so that $f$ is separately continuous on $X \times Y_{\infty}$. Let $\tau$ be the topology of pointwise convergence on $\mathcal{C}(X)$. Since the map

$$
Y_{\infty} \rightarrow \mathcal{C}(X), \quad y \mapsto f(\cdot, y)
$$


is continuous with respect to the given topology on $Y_{\infty}$ and to $\tau$ on $\mathcal{C}(X)$, the set

$$
K:=\left\{f(\cdot, y): y \in Y_{\infty}\right\}
$$

is $\tau$-compact. By [11, Théorème 5], this means that $K$ is weakly compact, so that the weak topology and $\tau$ coincide on $K$. Let $\left(y_{\alpha}\right)_{\alpha}$ be a convergent net in $Y_{\infty}$ with limit $y$. Since $f\left(\cdot, y_{\alpha}\right) \stackrel{\tau}{\rightarrow} f(\cdot, y)$, it follows that

$$
\lim _{\alpha} \int_{X} f\left(x, y_{\alpha}\right) d \mu(x)=\int_{X} f(x, y) d \mu(x) .
$$

This means that the function

$$
Y_{\infty} \rightarrow \mathbb{C}, \quad y \mapsto \int_{X} f(x, y) d \mu(x)
$$

is continuous on $Y_{\infty}$; since it vanishes at $\infty$ by definition, this establishes (i).

Remark For compact spaces, Lemma 2.2 is well known ([3, Theorem A.20]).

Lemma 2.3 Let $X$ and $Y$ be locally compact Hausdorff spaces, and let $f \in \mathcal{S C}_{0}(X \times Y)$. Then the bilinear map

$$
\Phi_{f}: M(X) \times M(Y) \rightarrow \mathbb{C}, \quad(\mu, \nu) \mapsto \int_{Y} \int_{X} f(x, y) d \mu(x) d \nu(y)
$$

belongs to $\mathcal{L}_{w^{*}}(M(X), M(Y) ; \mathbb{C})$.

Proof Clearly, $\Phi_{f}$ is bounded, and it is immediate from Lemma 2.2(i) that it is $w^{*}$ continuous in the second variable. Since $f$ is separately continuous, it is $\mu \otimes \nu$-measurable for all $\mu \in M(X)$ and $\nu \in M(Y)$ by [15]. It follows that the integral in (2) not only exists, but — by Fubini's theorem — is independent of the order of integration, i.e.

$$
\Phi_{f}(\mu, \nu)=\int_{X} \int_{Y} f(x, y) d \nu(y) d \mu(x) \quad(\mu \in M(X), \nu \in M(Y)) .
$$

It then follows from Lemma 2.2(ii) that $\Phi_{f}$ is also $w^{*}$-continuous in the first variable.

Proposition 2.4 Let $X$ and $Y$ be locally compact Hausdorff spaces. Then

$$
\mathcal{S C}_{0}(X \times Y) \rightarrow \mathcal{L}_{w^{*}}^{2}(M(X), M(Y) ; \mathbb{C}), \quad f \mapsto \Phi_{f}
$$

is an isometric isomorphism. 
Proof Clearly, $\left\|\Phi_{f}\right\| \leq\|f\|_{\infty}$ for all $f \in \mathcal{S C}_{0}(X \times Y)$. On the other hand,

$$
\begin{aligned}
\left\|\Phi_{f}\right\| & \geq \sup \left\{\left|\Phi_{f}\left(\delta_{x}, \delta_{y}\right)\right|: x \in X, y \in Y\right\} \\
& =\sup \{|f(x, y)|: x \in X, y \in Y\} \\
& =\|f\|_{\infty} \quad\left(f \in \mathcal{S C}_{0}(X \times Y)\right),
\end{aligned}
$$

so that (邽) is an isometry.

Let $\Phi \in \mathcal{L}_{w^{*}}(M(X), M(Y) ; \mathbb{C})$ be arbitrary, and define

$$
f: X \times Y \rightarrow \mathbb{C}, \quad(x, y) \mapsto \Phi\left(\delta_{x}, \delta_{y}\right)
$$

It is immediate that $f \in \mathcal{S C}_{0}(X \times Y)$ such that $\Phi_{f}\left(\delta_{x}, \delta_{y}\right)=\Phi\left(\delta_{x}, \delta_{y}\right)$ for all $x \in X$ and $y \in Y$. Separate $w^{*}$-continuity yields that $\Phi=\Phi_{f}$.

We shall, from now on, identify $\mathcal{S C}_{0}(X \times Y)$ and $\mathcal{L}_{w^{*}}(M(X), M(Y) ; \mathbb{C})$ as Banach spaces.

Proposition 2.5 Let $X$ and $Y$ be locally compact Hausdorff spaces. Then the map

$$
M(X \times Y) \rightarrow \mathcal{L}_{w^{*}}^{2}(M(X), M(Y) ; \mathbb{C})^{*}, \quad \mu \mapsto \Psi_{\mu}
$$

where

$$
\Psi_{\mu}(f):=\int_{X \times Y} f(x, y) d \mu(x, y) \quad\left(\mu \in M(X \times Y), f \in \mathcal{S C}_{0}(X \times Y)\right)
$$

is an isometry.

Proof By 15] again, the integral in (41) is well-defined. Since $\mathcal{C}_{0}(X \times Y) \subset \mathcal{S C}_{0}(X \times Y)$, it follows at once that $\left\|\Psi_{\mu}\right\|=\|\mu\|$ holds for all $\mu \in M(X \times Y)$.

\section{Separately $\mathcal{C}_{0}$-functions on locally compact groups}

Let $G$ and $H$ be locally compact groups. Then $\mathcal{S C}_{0}(G \times H)$ becomes a Banach $M(H)$ $M(G)$-bimodule through the following convolution formulae for $f \in \mathcal{S C}_{0}(G \times H), \mu \in$ $M(H)$, and $\nu \in M(G)$ :

$$
(\mu \cdot f)(g, h):=\int_{H} f(g, h k) d \mu(k) \quad(g \in G, h \in H)
$$

and

$$
(f \cdot \nu)(g, h):=\int_{G} f(k g, h) d \nu(k) \quad(g \in G, h \in H) .
$$

The following extension of Proposition 2.4 is then routinely checked: 
Proposition 3.1 Let $G$ and $H$ be locally compact groups. Then

$$
\mathcal{S C}_{0}(G \times H) \rightarrow \mathcal{L}_{w^{*}}^{2}(M(G), M(H) ; \mathbb{C}), \quad f \mapsto \Phi_{f}
$$

as defined in Proposition 2.4 is an isometric isomorphism of Banach $M(H)-M(G)$-bimodules.

Proof The $M(H)$ - $M(G)$-bimodule action on $\mathcal{S C}_{0}(G \times H)$ induces an $M(G)$ - $M(H)$-bimodule action on $\mathcal{S C}_{0}(G \times H)^{*}$. Embedding $M(G) \hat{\otimes} M(H)$ into $\mathcal{S C}_{0}(G \times H)^{*}$, we need to show that $M(G) \hat{\otimes} M(H)$ is a $M(G) \hat{\otimes} M(H)$-submodule of $\mathcal{S C}_{0}(G \times H)^{*}$ such that the module actions are the canonical ones (see (11)).

Let $\kappa, \mu \in M(G)$, and let $\nu \in M(H)$. Then we have for $f \in \mathcal{S C}_{0}(G \times H)$ :

$$
\begin{aligned}
\langle f, \kappa \cdot(\mu \otimes \nu)\rangle & =\langle f \cdot \kappa, \mu \otimes \nu\rangle \\
& =\int_{H} \int_{G} \int_{G} f(k g, h) d \kappa(k) d \mu(g) d \nu(h) \\
& =\int_{H} \int_{G} f(g, h) d(\kappa * \mu)(g) d \nu(h) \\
& =\langle f, \kappa * \mu \otimes \nu\rangle .
\end{aligned}
$$

An analogous property holds for the right $M(H)$-module action on $\mathcal{S C}_{0}(G \times H)^{*}$.

Remark It is easy to see that $\mathcal{C}_{0}(G \times H)$ is a closed $M(H)-M(G)$-submodule of $\mathcal{S C}_{0}(G \times$ $H)$, so that $M(G \times H) \cong \mathcal{C}_{0}(G \times H)^{*}$ is a quotient of $\mathcal{S C}_{0}(G \times H)^{*}$. It is easily checked that

$$
\mu \cdot \nu=\left(\mu \otimes \delta_{e}\right) * \nu \quad(\mu \in M(G), \nu \in M(G \times H))
$$

and

$$
\nu \cdot \mu=\nu *\left(\delta_{e} \otimes \mu\right) \quad(\mu \in M(H), \nu \in M(G \times H)) .
$$

We have the following:

Proposition 3.2 Let $G$ and $H$ be locally compact groups. Then:

(i) $M(G \times H)$ is a normal, dual Banach $M(G)-M(H)$-bimodule.

(ii) The map

$$
M(G \times H) \rightarrow \mathcal{L}_{w^{*}}^{2}(M(G), M(H) ; \mathbb{C})^{*}, \quad \mu \mapsto \Psi_{\mu},
$$

as defined in Proposition 2.5, is an isometric homomorphism of Banach $M(G)$ $M(H)$-bimodules. 
Proof The maps

$$
M(G) \rightarrow M(G \times H), \quad \mu \mapsto \mu \otimes \delta_{e}
$$

and

$$
M(H) \rightarrow M(G \times H), \quad \nu \mapsto \delta_{e} \otimes \nu
$$

are $w^{*}$-continuous. In view of the preceding remark and the fact that $M(G \times H)$ is a dual Banach algebra, (i) is immediate.

For (ii), let $\mu \in M(G)$ and $\nu \in M(G \times H)$. Then we have for any $f \in \mathcal{S C}_{0}(G \times H)$ :

$$
\begin{aligned}
\left\langle f, \mu \cdot \Psi_{\nu}\right\rangle & =\left\langle f \cdot \mu, \Psi_{\nu}\right\rangle \\
& =\int_{G \times H} \int_{G} f(k g, h) d \mu(k) d \nu(g, h) \\
& =\int_{G \times H} \int_{G \times H} f\left(k g, k^{\prime} h\right) d\left(\mu \otimes \delta_{e}\right)\left(k, k^{\prime}\right) d \nu(g, h) \\
& =\int_{G \times H} f(g, h) d\left(\left(\mu \otimes \delta_{e}\right) * \nu\right)(g, h) \\
& =\int_{G \times H} f(g, h) d(\mu \cdot \nu)(g, h) \\
& =\left\langle f, \Psi_{\mu \cdot \nu}\right\rangle .
\end{aligned}
$$

Hence, (6) is a left $M(G)$-module homomorphism.

Analogously, one shows that (6) is a right $M(H)$-module homomorphism.

With these preparations made, we can already give an alternative proof of 22, Proposition 5.2].

For any locally compact group $G$, the operator $\Delta_{*}:=\Delta_{M(G)}^{*} \mid \mathcal{C}_{0}(G)$ is given by

$$
\left(\Delta_{*} f\right)(g, h)=f(g h) \quad\left(f \in \mathcal{C}_{0}(G), g, h \in G\right) .
$$

If $G$ is compact, $\Delta_{*}$ maps $\mathcal{C}_{0}(G)=\mathcal{C}(G)$ into $\mathcal{C}(G \times G)=\mathcal{C}_{0}(G \times G)$. Hence, $\Delta_{w^{*}}$ drops to an $M(G)$-bimodule homomorphism $\Delta_{0, w^{*}}: M(G \times G) \rightarrow M(G)$.

Proposition 3.3 Let $G$ be a compact group. Then there is a normal, virtual diagonal for $M(G)$.

Proof Since $G$ is amenable, $M(G)$ is Connes-amenable (this is the easy direction of Theorem 5.3).

Define a $w^{*}$-continuous derivation

$$
D: M(G) \rightarrow M(G \times G), \quad \mu \mapsto \mu \otimes \delta_{e}-\delta_{e} \otimes \mu .
$$


It is immediate that $D$ attains its values in ker $\Delta_{0, w^{*}}$. Being the kernel of a $w^{*}$-continuous $M(G)$-bimodule homomorphism between normal, dual Banach $M(G)$-bimodules, ker $\Delta_{0, w^{*}}$ is a normal, dual Banach $M(G)$-bimodule in its own right. Since $M(G)$ is Connes-amenable, there is $\mathrm{N} \in \operatorname{ker} \Delta_{0, w^{*}}$ such that

$$
D \mu=\mu \cdot \mathrm{N}-\mathrm{N} \cdot \mu \quad(\mu \in M(G)) .
$$

Letting $\mathrm{M}:=\delta_{e} \otimes \delta_{e}-\mathrm{N}$, and embedding $\mathrm{M}$ into $\mathcal{S C}_{0}(G \times G)^{*}$ via Proposition 3.2, we obtain a normal, virtual diagonal for $M(G)$.

Remark The proof of Proposition 3.3, does not carry over to non-compact, locally compact groups with Connes-amenable measure algebra because, for non-compact $G$, we no longer have $\Delta_{*} \mathcal{C}_{0}(G) \subset \mathcal{C}_{0}(G \times G)$; in fact, it is easy to see that $\Delta_{*} \mathcal{C}_{0}(G) \cap \mathcal{C}_{0}(G \times G)=\{0\}$ whenever $G$ is not compact.

\section{A left introverted subspace of separately $\mathcal{C}_{0}$-functions}

For general, possibly non-compact, locally compact groups, we need a Banach $M(G)$ bimodule that can play the rôle of $M(G \times G)$ in the proof of Proposition 3.3.

Let $G$ be a locally compact group. For a function $f: G \rightarrow \mathbb{C}$ and for $g \in G$, define functions $L_{g} f, R_{g} f: G \rightarrow \mathbb{C}$ through

$$
\left(L_{g} f\right)(h):=f(g h) \quad \text { and } \quad\left(R_{g} f\right)(h):=f(h g) \quad(h \in G) .
$$

A closed subspace $E$ of $\ell^{\infty}(G)$ is called left invariant if $L_{g} f \in E$ for each $f \in E$ and $g \in G$. A left invariant subspace $E$ of $\ell^{\infty}(G)$ is called left introverted if, for each $\phi \in E^{*}$, the function

$$
\phi \bullet f: G \rightarrow \mathbb{C}, \quad g \mapsto\left\langle L_{g} f, \phi\right\rangle
$$

belongs again to $E$.

Examples $\quad$ 1. $\ell^{\infty}(G)$ is trivially left introverted.

2. $\mathcal{C}_{0}(G)$ is left introverted ([14, (19.5) Lemma]).

3. The space

$$
\mathcal{L U C}(G):=\left\{f \in \mathcal{C}_{b}(G): G \ni g \mapsto L_{g} f \text { is norm continuous }\right\}
$$

of left uniformly continuous functions on $G$ is left introverted (21, (2.11) Proposition]). 
If $E$ is a left introverted subspace of $\ell^{\infty}(G)$, then $E^{*}$ is a Banach algebra in a natural manner:

$$
\langle\phi * \psi, f\rangle:=\langle\psi \bullet f, \phi\rangle \quad\left(\phi, \psi \in E^{*}, f \in E\right) .
$$

In case $E=\mathcal{C}_{0}(G)$, this is the usual convolution product on $M(G)$.

We now define a certain space of separately $\mathcal{C}_{0}$-functions which is, as we shall see, left introverted. For any locally compact group $G$, let $G_{\mathcal{L U C}}$ denote the character space of the commutative $C^{*}$-algebra $\mathcal{L U C}(G)$. The multiplication $*$ on $\mathcal{L U C}(G)^{*}$ turns $G_{\mathcal{L U C}}$ into a compact semigroup with continuous right multiplication that contains $G$ as a dense subsemigroup (匹1). Also, we use $G^{\text {op }}$ to denote the same group, but with reversed multiplication.

Definition 4.1 For locally compact groups $G$ and $H$, let

$$
\begin{aligned}
& \mathcal{L U C S C}_{0}\left(G \times H^{\mathrm{op}}\right) \\
& :=\left\{f \in \mathcal{L U C}\left(G \times H^{\mathrm{op}}\right): \omega \bullet f \in \mathcal{S C}_{0}(G \times H) \text { for all } \omega \in\left(G \times H^{\mathrm{op}}\right)_{\mathcal{L U C}}\right\} .
\end{aligned}
$$

Remark If both $G$ and $H$ are compact, then $\mathcal{L U C S C}_{0}\left(G \times H^{\text {op }}\right)=\mathcal{C}(G \times H)$.

Lemma 4.2 Let $G$ and $H$ be locally compact groups, let $f \in \mathcal{L U C S C}_{0}\left(G \times H^{\mathrm{op}}\right)$, and let $h \in H$. Then $\left\{L_{(g, h)} f: g \in G\right\}$ is relatively weakly compact.

Proof The claim is clear for compact $G$, so that we may suppose without loss of generality that $G$ is not compact.

By [11, Théorème 5], it is sufficient to show that $\left\{L_{(g, h)} f: g \in G\right\}$ is relatively compact in $\mathcal{L U C}\left(G \times H^{\mathrm{op}}\right)$ with respect to the topology of pointwise convergence on $\left(G \times H^{\mathrm{op}}\right) \mathcal{L U C}$. Also, we may suppose without loss of generality that $h=e$.

Let $\hat{f} \in \mathcal{C}\left(\left(G \times H^{\mathrm{op}}\right)_{\mathcal{L U C}}\right)$ denote the Gelfand transform of $f$. The map

$$
G \rightarrow \mathbb{C}, \quad g \mapsto \hat{f}\left(\left(\delta_{g} \otimes \delta_{e}\right) * \omega\right)
$$

is continuous for each $\omega \in\left(G \times H^{\mathrm{op}}\right)_{\mathcal{L U C}}$. Let $G_{\infty}$ denote the one-point-compactification of $G$. Let $\left(g_{\alpha}\right)_{\alpha}$ be a net in $G$ with $g_{\alpha} \rightarrow \infty$. For any $\omega \in\left(G \times H^{\mathrm{op}}\right)_{\mathcal{L U C}}$, we then have

$$
\hat{f}\left(\left(\delta_{g_{\alpha}} \otimes \delta_{e}\right) * \omega\right)=(\omega \bullet f)\left(g_{\alpha}, e\right) \rightarrow 0
$$

because $\omega \bullet f \in \mathcal{S C}_{0}(G \times H)$. Hence,

$$
G \rightarrow \mathcal{L U C}\left(G \times H^{\mathrm{op}}\right), \quad g \mapsto L_{(g, e)} f
$$

extends as a continuous map to $G_{\infty}$, where $\mathcal{L U C}\left(G \times H^{\text {op }}\right)$ is equipped with the topology of pointwise convergence on $\left(G \times H^{\mathrm{op}}\right) \mathcal{L U C}$. As the continuous image of the compact space $G_{\infty}$, the range of (7) is compact in the topology of pointwise convergence on $\left(G \times H^{\mathrm{op}}\right) \mathcal{L U C}$. 
Proposition 4.3 Let $G$ and $H$ be locally compact groups. Then $\mathcal{L U C S C}_{0}\left(G \times H^{\mathrm{op}}\right)$ is left introverted.

Proof Let $f \in \mathcal{L U C S C}_{0}\left(G \times H^{\mathrm{op}}\right)$, and let $\phi \in \mathcal{L U C S C}_{0}\left(G \times H^{\mathrm{op}}\right)^{*}$. Since $\mathcal{L U C}\left(G \times H^{\mathrm{op}}\right)$ is left introverted, it is immediate that $\phi \bullet f \in \mathcal{L U C}\left(G \times H^{\mathrm{op}}\right)$.

We first claim that $\phi \bullet f \in \mathcal{S C}_{0}(G \times H)$.

Fix $h \in H$; we will show that $(\phi \bullet f)(\cdot, h)$, i.e. the function

$$
G \rightarrow \mathbb{C}, \quad g \mapsto\left\langle L_{(g, h)} f, \phi\right\rangle
$$

belongs to $\mathcal{C}_{0}(G)$. Since $(\phi \bullet f)(\cdot, h)$ is clearly continuous, all we have to show is that it vanishes at $\infty$. Suppose without loss of generality that $G$ is not compact, and let $\left(g_{\alpha}\right)_{\alpha}$ be a net in $G$ such that $g_{\alpha} \rightarrow \infty$. Let $\tau$ denote the topology of pointwise convergence on $G \times H$. It is clear that $L_{\left(g_{\alpha}, h\right)} f \stackrel{\tau}{\rightarrow} 0$. Since $\left\{L_{(g, h)} f: g \in G\right\}$ is relatively weakly compact by Lemma 4.2, the weak topology and $\tau$ coincide on the weak closure of $\left\{L_{(g, h)} f: g \in G\right\}$, so that, in particular, $\left\langle L_{\left(g_{\alpha}, h\right)} f, \phi\right\rangle \rightarrow 0$.

Analogously, one sees that $(\phi \bullet f)(g, \cdot) \in \mathcal{C}_{0}(H)$ for each $g \in G$.

Let $\omega \in\left(G \times H^{\mathrm{op}}\right) \mathcal{L U C}$. Since, by the foregoing,

$$
\omega \bullet(\phi \bullet f)=(\omega * \phi) \bullet f \in \mathcal{S C}_{0}(G \times H),
$$

it follows that $\phi \bullet f \in \mathcal{L U C S C}_{0}\left(G \times H^{\text {op }}\right)$.

Theorem 4.4 Let $G$ and $H$ be locally compact groups. Then we have:

(i) $\mathcal{L U C S C}_{0}\left(G \times H^{\mathrm{op}}\right)$ is a closed $M(H)-M(G)$-submodule of $\mathcal{S C}_{0}\left(G \times H^{\mathrm{op}}\right)$.

(ii) $\mathcal{L U C S C}_{0}\left(G \times H^{\mathrm{op}}\right)^{*}$ is a normal, dual Banach $M(G)-M(H)$-bimodule.

(iii) If $H=G$, then $\Delta_{*}$ maps $\mathcal{C}_{0}(G)$ into $\mathcal{L U C S C}_{0}\left(G \times G^{\mathrm{op}}\right)$.

Proof For (i), first note that it is routinely checked that $\mu \cdot f, f \cdot \nu \in \mathcal{L U C}\left(G \times H^{\text {op }}\right)$ for all $f \in \mathcal{L U C S C}_{0}\left(G \times H^{\mathrm{op}}\right)$ and all $\mu \in M(G)$ and $\nu \in M(H)$. Fix $f \in \mathcal{L U C S C}_{0}\left(G \times H^{\mathrm{op}}\right)$, $\mu \in M(G), \nu \in M(H)$, and let $\omega \in\left(G \times H^{\text {op }}\right)_{\mathcal{L U C}}$. Since

$$
\omega \bullet(\mu \cdot f)(g, h)=\left\langle\mu \cdot L_{(g, h)} f, \omega\right\rangle \quad\left((g, h) \in G \times H^{\mathrm{op}}\right),
$$

an application of Lemma 4.2 as in the proof of Proposition 4.3 yields that $\omega \bullet(\mu \cdot f) \in$ $\mathcal{S C}_{0}\left(G \times H^{\text {op }}\right)$. A similar, but easier argument yields that $\omega \bullet(f \cdot \nu) \in \mathcal{S C}_{0}\left(G \times H^{\text {op }}\right)$.

For (ii), first observe that the canonical embedding of $M\left(G \times H^{\mathrm{op}}\right)$ into $\mathcal{L U C}\left(G \times H^{\mathrm{op}}\right)^{*}$ via integration is an algebra homomorphism. If we view $M\left(G \times H^{\text {op }}\right)$ as a $M(G)-M(H)$ submodule of $\mathcal{L U C S C}_{0}\left(G \times H^{\mathrm{op}}\right)^{*}$ (through Proposition 3.2(ii)), we see routinely that

$$
\mu \cdot \nu=\left.\left(\mu \otimes \delta_{e}\right) * \nu\right|_{\mathcal{L U C S C}_{0}(G \times H)} \quad\left(\mu \in M(G), \nu \in M\left(G \times H^{\mathrm{op}}\right)\right) .
$$


Fix $\mu \in M(G)$. By (the simple direction of) [19] - actually already proven in [26] -, the map

$$
\mathcal{L U C}\left(G \times H^{\mathrm{op}}\right)^{*} \rightarrow \mathcal{L U C}\left(G \times H^{\mathrm{op}}\right)^{*}, \quad \phi \mapsto\left(\mu \otimes \delta_{e}\right) * \phi
$$

is $w^{*}$-continuous. Let $\phi \in \mathcal{L U C}\left(G \times H^{\mathrm{op}}\right)^{*}$ be arbitrary, and choose a net $\left(\nu_{\alpha}\right)_{\alpha}$ in $M\left(G \times H^{\mathrm{op}}\right)$ that converges to $\phi$ in the $w^{*}$-topology (the existence of such a net follows with a simple Hahn-Banach argument). Then (8) and the $w^{*}$-continuity of (9), yield that

$$
\mu \cdot \phi=w^{*}-\lim _{\alpha} \mu \cdot \nu_{\alpha}=w^{*}-\lim _{\alpha}\left(\mu \otimes \delta_{e}\right) * \nu_{\alpha}=\left(\mu \otimes \delta_{e}\right) * \phi .
$$

Let $f \in \mathcal{L U C S C}_{0}\left(G \times H^{\text {op }}\right)$. Then we have

$$
\langle f, \mu \cdot \phi\rangle=\left\langle f,\left(\mu \otimes \delta_{e}\right) * \phi\right\rangle=\left\langle\phi \bullet f, \mu \otimes \delta_{e}\right\rangle .
$$

By Proposition 4.3, $\mathcal{L U C S C}_{0}\left(G \times H^{\text {op }}\right)$ is left introverted, so that, in particular, $\phi \bullet f \in$ $\mathcal{S C}_{0}\left(G \times H^{\mathrm{op}}\right)$. Let $\left(\mu_{\alpha}\right)_{\alpha}$ be a net in $M(G)$ that converges to $\mu$ in the $w^{*}$-topology. Then (10) yields:

$$
\begin{aligned}
\lim _{\alpha}\left\langle f, \mu_{\alpha} \cdot \phi\right\rangle & =\lim _{\alpha}\left\langle\mu_{\alpha} \otimes \delta_{e}, \phi \bullet f\right\rangle \\
& =\lim _{\alpha}\left\langle(\phi \bullet f)(\cdot, e), \mu_{\alpha}\right\rangle \\
& =\langle(\phi \bullet f)(\cdot, e), \mu\rangle \\
& =\left\langle\phi \bullet f, \mu \otimes \delta_{e}\right\rangle \\
& =\langle f, \mu \cdot \phi\rangle .
\end{aligned}
$$

It follows that, for any $\phi \in \mathcal{L U C S C}_{0}\left(G \times H^{\text {op }}\right)^{*}$, the map

$$
M(G) \rightarrow \mathcal{L U C S C}_{0}\left(G \times H^{\mathrm{op}}\right)^{*}, \quad \mu \mapsto \mu \cdot \phi
$$

is $w^{*}$-continuous. Noting that

$$
\phi \cdot \nu=\phi *\left(\delta_{e} \otimes \nu\right) \quad(\nu \in M(H)),
$$

we see analoguously that

$$
M(H) \rightarrow \mathcal{L U C S C}_{0}\left(G \times H^{\mathrm{op}}\right)^{*}, \quad \nu \mapsto \phi \cdot \nu
$$

is $w^{*}$-continuous for all $\phi \in \mathcal{L U C S C}_{0}\left(G \times H^{\mathrm{op}}\right)^{*}$. This proves (ii).

Suppose that $H=G$. It is well known $\mathcal{C}_{0}(G) \subset \mathcal{L U C}(G) \cap \mathcal{R U C}(G)$, where

$$
\mathcal{R U C}(G):=\left\{f \in \mathcal{C}_{b}(G): G \ni g \mapsto R_{g} f \text { is norm continuous }\right\} .
$$

Let $f \in \mathcal{C}_{0}(G)$, and note that

$$
L_{(g, h)} \Delta_{*} f=\Delta_{*}\left(L_{g} R_{h} f\right) \quad\left((g, h) \in G \times G^{\mathrm{op}}\right) .
$$


The norm continuity of $\Delta_{*}$ shows that $\Delta_{*} f \in \mathcal{L U C}\left(G \times G^{\text {op }}\right)$. To show that $\Delta_{*} f \in$ $\mathcal{L U C S C}_{0}\left(G \times G^{\text {op }}\right)$, let $\omega \in\left(G \times G^{\text {op }}\right)_{\mathcal{L U C}}$. Let $\left(\left(g_{\alpha}, h_{\alpha}\right)\right)_{\alpha}$ be a net in $G \times G^{\text {op }}$ such that $\left(g_{\alpha}, h_{\alpha}\right) \rightarrow \omega$. Passing to a subnet, we may suppose that $\left(g_{\alpha} h_{\alpha}\right)_{\alpha}$ converges to some $k \in G$ or tends to infinity. In the first case, we have

$$
\left(\omega \bullet \Delta_{*} f\right)(g, h)=\lim _{\alpha} \Delta_{*} f\left(g g_{\alpha}, h_{\alpha} h\right)=\lim _{\alpha} f\left(g g_{\alpha} h_{\alpha} h\right)=f(g k h) \quad\left((g, h) \in G \times H^{\mathrm{op}}\right)
$$

and in the second one

$$
\left(\omega \bullet \Delta_{*} f\right)(g, h)=\lim _{\alpha} \Delta_{*} f\left(g g_{\alpha}, h_{\alpha} h\right)=\lim _{\alpha} f\left(g g_{\alpha} h_{\alpha} h\right)=0 \quad\left((g, h) \in G \times H^{\mathrm{op}}\right) .
$$

In either case, $\omega \bullet \Delta_{*} f \in \mathcal{S C}_{0}\left(G \times H^{\text {op }}\right)$ holds. This proves (iii).

\section{Connes-amenability of $M(G)$}

Let $G$ be a locally compact group. As a consequence of Theorem 4.4(iii), $\Delta_{M(G)}$ extends to an $M(G)$-bimodule homomorphism $\Delta_{0, w^{*}}: \mathcal{L U C S C}_{0}\left(G \times G^{\text {op }}\right)^{*} \rightarrow M(G)$ :

Proposition 5.1 Let $G$ be a locally compact group such that $M(G)$ is Connes-amenable. Then there is $\mathrm{M} \in \mathcal{L U C S C}_{0}\left(G \times G^{\mathrm{op}}\right)^{*}$ such that

$$
\mu \cdot \mathrm{M}=\mathrm{M} \cdot \mu \quad(\mu \in M(G)) \quad \text { and } \quad \Delta_{0, w^{*}} \mathrm{M}=\delta_{e} .
$$

Proof Define a derivation

$$
D: M(G) \rightarrow \mathcal{L U C S C}_{0}\left(G \times G^{\mathrm{op}}\right)^{*}, \quad \mu \mapsto \mu \otimes \delta_{e}-\delta_{e} \otimes \mu .
$$

It is easy to see that $D$ is $w^{*}$-continuous and attains its values in ker $\Delta_{0, w^{*}}$. Being the kernel of a $w^{*}$-continuous bimodule homomorphism, ker $\Delta_{0, w^{*}}$ is a $w^{*}$-closed submodule of the normal, dual Banach $M(G)$-module $\mathcal{L U C S C}_{0}\left(G \times G^{\text {op }}\right)^{*}$ and thus a normal, dual Banach $M(G)$-module in its own right. Since $M(G)$ is Connes-amenable, there is thus $\mathrm{N} \in \operatorname{ker} \Delta_{0, w^{*}}$ such that

$$
D \mu=\mu \cdot \mathrm{N}-\mathrm{N} \cdot \mu \quad(\mu \in M(G)) .
$$

The element

$$
\mathrm{M}:=\delta_{e} \otimes \delta_{e}-\mathrm{N}
$$

then has the desired properties.

Remark Since $\mathcal{L U C S C}_{0}(G \times G)^{*}$ is only a quotient of $\mathcal{S C}_{0}(G \times G)^{*}$, Proposition 5.1 does not allow us to conclude that $M(G)$ has a normal, virtual diagonal. 
Lemma 5.2 Let $G$ and $H$ be locally compact groups. Then $\mathcal{L U C S C}_{0}\left(G \times H^{\mathrm{op}}\right)$ is an essential ideal of $\mathcal{L U C}\left(G \times H^{\mathrm{op}}\right)$.

Proof Let $f \in \mathcal{L U C S C}_{0}\left(G \times H^{\text {op }}\right)$, let $F \in \mathcal{L U C}\left(G \times H^{\text {op }}\right) \subset \mathcal{L U C}\left(G \times H^{\text {op }}\right)$, and let $\omega \in\left(G \times H^{\text {op }}\right)_{\mathcal{L U C}}$. Let $\left(\left(g_{\alpha}, h_{\alpha}\right)\right)_{\alpha}$ be a net in $G \times H^{\text {op }}$ converging to $\omega$. Since

$$
\omega \bullet(f F)=\lim _{\alpha} R_{\left(g_{\alpha}, h_{\alpha}\right)}(f F)=\lim _{\alpha}\left(R_{\left(g_{\alpha}, h_{\alpha}\right)} f\right)\left(R_{\left(g_{\alpha}, h_{\alpha}\right)} F\right)=(\omega \bullet f)(\omega \bullet F)
$$

with pointwise convergence on $G \times H$ and since $\omega \bullet F \in \mathcal{L U C}\left(G \times H^{\mathrm{op}}\right)$, it follows that $\omega \bullet(f F) \in \mathcal{S C}_{0}(G \times H)$. Hence, $\mathcal{L U C S C}_{0}\left(G \times H^{\mathrm{op}}\right)$ is an ideal of $\mathcal{L U C}\left(G \times H^{\mathrm{op}}\right)$. Since $\mathcal{C}_{0}(G \times H) \subset \mathcal{L U C S C}_{0}\left(G \times H^{\text {op }}\right)$, it is even an essential ideal.

Theorem 5.3 For a locally compact group $G$, the following are equivalent:

(i) $G$ is amenable.

(ii) $M(G)$ is Connes-amenable.

Proof (i) $\Longrightarrow$ (ii): By [16, Theorem 2.5], $L^{1}(G)$ is amenable. Since $L^{1}(G)$ is $w^{*}$-dense in $M(G)$, [22, Proposition 4.2] yields the Connes-amenability of $M(G)$.

(ii) $\Longrightarrow$ (i): Let $\mathrm{M} \in \mathcal{L U C S C}_{0}\left(G \times G^{\text {op }}\right)^{*}$ be as in Proposition 5.1. View $\mathrm{M}$ as a measure on the character space of the commutative $C^{*}$-algebra $\mathcal{L U C S C}_{0}\left(G \times G^{\text {op }}\right)$, so that $|\mathrm{M}| \in \mathcal{L U C S C}_{0}\left(G \times G^{\text {op }}\right)^{*}$ can be defined in terms of measure theory. It is routinely checked that $|\mathrm{M}| \neq 0$, and

$$
\delta_{g} \cdot|\mathrm{M}|=|\mathrm{M}| \cdot \delta_{g} \quad(g \in G)
$$

By Lemma $5.2 \mathcal{L U C S C}_{0}\left(G \times G^{\text {op }}\right)$ is an essential, closed ideal in $\mathcal{L U C}\left(G \times G^{\text {op }}\right)$. We may therefore view $\mathcal{L U C}\left(G \times G^{\text {op }}\right)$ as a $C^{*}$-subalgebra of the multiplier algebra $\mathcal{M}\left(\mathcal{L U C S C}_{0}(G \times\right.$ $\left.\left.G^{\mathrm{op}}\right)\right)$. Since $\mathcal{M}\left(\mathcal{L U C S C}_{0}\left(G \times G^{\mathrm{op}}\right)\right)$, in turn, embeds canonically into $\mathcal{L U C S C}_{0}\left(G \times G^{\mathrm{op}}\right)^{* *}$, we may view $\mathcal{M}\left(\mathcal{L U C S C}_{0}\left(G \times G^{\text {op }}\right)\right)$ and thus $\mathcal{L U C}\left(G \times G^{\text {op }}\right)$ as a $C^{*}$-subalgebra of $\mathcal{L U C S C}_{0}\left(G \times G^{\mathrm{op}}\right)^{* *}$, so that, in particular, $\langle f,|\mathrm{M}|\rangle$ is well-defined for each $f \in \mathcal{L U C}(G \times$ $\left.G^{\text {op }}\right)$. Note that the embedding of $\mathcal{L U C}\left(G \times G^{\text {op }}\right)$ into $\mathcal{L U C S C}_{0}\left(G \times G^{\text {op }}\right)^{\text {** }}$ is an $M(G)$ bimodule homormorphism (where the $M(G)$-bimodule action on $\mathcal{L U C}\left(G \times G^{\text {op }}\right.$ ) is defined as on $\left.\mathcal{S C}_{0}(G \times G)\right)$. Define

$$
m: \mathcal{L U C}(G) \rightarrow \mathbb{C}, \quad f \mapsto\langle f \otimes 1,|\mathrm{M}|\rangle
$$

Since $f \otimes 1 \in \mathcal{L U C}\left(G \times G^{\text {op }}\right)$ for each $f \in \mathcal{L U C}(G), m$ is a well-defined, positive, linear 
functional. For $f \in \mathcal{L U C}(G)$ and $g \in G$, we have:

$$
\begin{aligned}
\left\langle L_{g} f, m\right\rangle & =\left\langle L_{(g, e)}(f \otimes 1),|\mathrm{M}|\right\rangle \\
& =\left\langle f \otimes 1, \delta_{g} \cdot|\mathrm{M}|\right\rangle \\
& =\left\langle f \otimes 1,|\mathrm{M}| \cdot \delta_{g}\right\rangle \\
& =\left\langle L_{(e, g)}(f \otimes 1),|\mathrm{M}|\right\rangle \\
& =\langle f \otimes 1,|\mathrm{M}|\rangle \\
& =\langle f, m\rangle .
\end{aligned}
$$

Normalizing $m$, we thus obtain a left invariant mean on $\mathcal{L U C}(G)$. Hence, $G$ is amenable by [23, Theorem 1.1.9].

We believe that assertions (i) and (ii) in Theorem 5.3 are equivalent to:

(iii) $M(G)$ has a normal virtual diagonal.

Although we have been unable to prove this, Proposition 3.3 as well as the following corollary support this belief:

Corollary 5.4 Let $G$ be a discrete group. Then the following are equivalent:

(i) $G$ is amenable.

(ii) $\ell^{1}(G)$ is Connes-amenable.

(iii) There is a normal, virtual diagonal for $\ell^{1}(G)$.

Proof (i) $\Longrightarrow$ (iii): If $G$ is amenable, $\ell^{1}(G)$ is amenable, so that there is a virtual diagonal $\mathrm{M} \in\left(\ell^{1}(G) \hat{\otimes} \ell^{1}(G)\right)^{* *}$ for $\ell^{1}(G)$. Let $\rho:\left(\ell^{1}(G) \hat{\otimes} \ell^{1}(G)\right)^{* *} \rightarrow \mathcal{L}_{w^{*}}^{2}\left(\ell^{1}(G), \mathbb{C}\right)^{*}$ denote the restriction map. Then $\rho(\mathrm{M})$ is a normal, virtual diagonal for $\ell^{1}(G)$.

Since (i) $\Longleftrightarrow$ (ii) by Theorem 5.3 , and since (iii) $\Longrightarrow$ (ii) for any dual Banach algebra, this proves the corollary.

Remark Since discrete groups are trivially inner amenable, the equivalence of (i) and (ii) in Corolllary [5.4 can alternatively be deduced from [20]: If $\ell^{1}(G)$ is Connes-amenable, then so is $\operatorname{VN}(G)$ by [22, Proposition 4.2], which, by [20], establishes the amenability of G.

\section{References}

[1] J. F. Berglund, H. D. Junghenn, and P. Milnes, Analysis on Semigroups. WileyInterscience, 1988. 
[2] J. W. Bunce and W. L. PAschke, Quasi-expectations and amenable von Neumann algebras. Proc. Amer. Math. Soc. 71 (1978), 232-236.

[3] R. B. Burckel, Weakly Almost Periodic Functions on Semigroups. Gordon and Breach, 1970.

[4] A. Connes, Classification of injective factors. Ann. of Math. 104 (1976), 73-114.

[5] A. Connes, On the cohomology of operator algebras. J. Funct. Anal. 28 (1978), 248-253.

[6] G. Corach and J. E. Galé, Averaging with virtual diagonals and geometry of representations. In: E. Albrecht and M. Mathieu (ed.s), Banach Algebras '97, pp. 87-100. Walter de Grutyer, 1998.

[7] H. G. Dales, F. Ghahramani, and A. Ya. HelemskiI, The amenability of measure algebras. J. London Math. Soc. (to appear).

[8] E. G. Effros, Amenability and virtual diagonals for von Neumann algebras. J. Functional Analysis 78 (1988), 137-153.

[9] E. G. Effros and E. C. Lance, Tensor products of operator algebras. J. Funct. Anal. 25 (1977), 1-34.

[10] E. G. Effros and A. Kishimoto, Module maps and Hochschild-Johnson cohomology. Indiana Univ. Math. J. 36 (1987), 257-276.

[11] A. Grothendieck, Critères de compacité dans les espaces fonctionnels généraux. Amer. J. Math. 74 (1953), 168-186.

[12] U. HaAgerup, All nuclear $C^{*}$-algebras are amenable. Invent. math. 74 (1983), 305-319.

[13] A. Ya. HelemskiI , Homological essence of amenability in the sense of A. Connes: the injectivity of the predual bimodule (translated from the Russion). Math. USSR-Sb 68 (1991), $555-566$.

[14] E. Hewitt and K. A. Ross, Abstract Harmonic Analysis, I and II. Springer Verlag, 1979.

[15] B. E. Johnson, Separate continuity and measurability. Proc. Amer. Math. Soc. 20 (1969), $420-422$.

[16] B. E. Johnson, Cohomology in Banach algebras. Mem. Amer. Math. Soc. 127 (1972).

[17] B. E. Johnson, Approximate diagonals and cohomology of certain annihilator Banach algebras. Amer. J. Math. 94 (1972), 685-698.

[18] B. E. Johson, R. V. Kadison, and J. Ringrose, Cohomology of operator algebras, III. Bull. Soc. Math. France 100 (1972), 73-79.

[19] A. T.-M. LAU, Continuity of Arens multiplication on the dual space of bounded uniformly continuous functions on locally compact groups and topological semigroups. Math. Proc. Cambridge Phil. Soc. 99 (1986), 273-283. 
[20] A. T.-M. LAU and A. L. T. Paterson, Inner amenable locally compact groups. Trans. Amer. Math. Soc. 325 (1991), 155-169.

[21] A. L. T. Paterson, Amenability. American Mathematical Society, 1988.

[22] V. Runde, Amenability for dual Banach algebras. Studia Math. 148 (2001), 47-66.

[23] V. Runde, Lectures on Amenability. Lecture Notes in Mathematics 1774, Springer Verlag, 2001.

[24] S. Wassermann, On Tensor products of certain group $C^{*}$-algebras. J. Funct. Anal. 23 (1976), 239-254.

[25] S. Wassermann, Injective $W^{*}$-algebras. Math. Proc. Cambridge Phil. Soc. 82 (1977), 39-47.

[26] J. C. S. Wong, Invariant means on locally compact semigroups. Proc. Amer. Math. Soc. 31 (1972), 39-45.

[November 19, 2018]

Address: Department of Mathematical and Statistical Sciences

University of Alberta

Edmonton, Alberta

Canada T6G 2G1

E-mail: vrunde@ualberta.ca

URL: http://www.math.ualberta.ca/ runde/ 